\section{Tab. 1: Empfehlungen zur PORT beim inkomplett operierten NSCLC (Auszug)}

Leitlinie (Version)

NCCN, $2015[1]$

ESMO, 2013 [2]

ESMO, 2015 [3]

S3, $2011[4]$

Empfehlung (Stand 08/2015)

Stadien IIA/IIB (pT1-2 N1 oder pT3 N0)

R1: Re-Resektion + Chemotherapie oder CRT (simultan oder sequenziell) R2: Re-Resektion + Chemotherapie oder CRT (simultan empfohlen) Stadium IIIA (pT1-3 N2 oder pT3 N1)

R1: CRT (simultan oder sequenziell)

R2: CRT (simultan empfohlen)

R1 oder R2: PORT kann indiziert sein

R1/R2: PORT kann eine gute Option sein - Panelempfehlung

Stadium I/II: inkomplette Resektion + keine Nachresektion möglich: RT*

Stadium IIIA: inkomplette Resektion + keine Nachresektion möglich: RT*

PORT = postoperative Radiotherapie; $R T=$ Strahlentherapie; $C R T=$ Chemoradiotherapie; $N C C N=$ National Comprehen sive Cancer Network; ESMO = European Society for Medical Oncology; * keine Dosisangaben und keine Erläuterungen im weiteren Text; [1] NCCN-Guideline NSCLC, Version 07.2015; [2] Vansteenkiste J et al. Ann Oncol. 2013;24(Suppl 6):vi8998; [3] Eberhardt WE et al. Ann Oncol. 2015;26(8):1573-88; [4] Goeckenjan G et al. Pneumologie. 2011;65(8):e51-75.

bens nach 5 Jahren nicht profitieren konnten. Denn in dieser Arbeit lebten Patienten mit PORT signifikant länger und hatten eine bessere 5-Jahres-Überlebensrate als Patienten ohne PORT. Durch die Analyse wird die Wertigkeit einer PORT bei NSCLC-Patienten, Stadium II/III, mit R1/R2-Resektion gestützt. Möglicherweise kam es zu einer Selektion bei dem beschriebenen Patientenklientel:
Die bestrahlten Patienten waren häufiger jünger, hatten einen niedrigeren Nodalstatus und öfter eine zusätzliche Chemotherapie erhalten. Leider erfolgte keine zusätzliche Aufschlüsselung der Patienten nach R1oder R2-Situation. In mehreren Leitlinien wird die PORT bereits empfohlen (Tab. 1). Diese kann und sollte als gute Alternative zur erneuten Resektion angeboten werden.
Dabei sollte die Dosis zwischen 50-70 Gy betragen. Zum Teil sind hier etwas kontroverse Empfehlungen zu lesen: die alleinige Strahlentherapie, eine sequenzielle Radiochemotherapie oder gar eine simultane Radiochemotherapie werden als Optionen genannt. Für kombinierte Verfahren besteht sicherlich eine geringe Evidenz.

Eine intelligente Bestrahlungsplanung (mit Berücksichtigung der präoperativen Tumorausdehnung) und die Verwendung einer modernen, bildgeführten Strahlentherapie (IGRT, „image-guided radiotherapy“) machen das Verfahren effizient und sicher. Aus meiner Sicht sollten dennoch gewisse Parameter nach einem großen thoraxchirurgischen Eingriff kritisch geprüft werden: _ die postoperative Lungenfunktion,

- wichtige Komorbiditäten (v.a. schwerwiegende kardiale Erkrankungen),

_ eine adäquate postoperative Erholung und Wundheilung

_ Alter und Lebenserwartung.

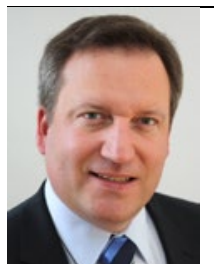

Dr. Thomas Schneider Strahlenzentrum Hamburg szhh.tom@gmail.com

\title{
NSCLC: EGFR-Mutationen machen Unterschied
}

\section{Jeder zehnte Patient mit nichtkleinzelligem Lungenkarzinom (NSCLC) weist nicht die typischen Mutationen des epidermalen Wachstumsfaktorrezeptors} (EGFR) auf. Ob diese Tumoren auch sensitiv für EGFR-Inhibitoren sind, ist unklar.

n einer Analyse von Daten aus 3 Studien mit Afatinib (Phase-II-Studie LUXLung-2 sowie Phase-III-Studien LUXLung 3 and LUX-Lung 6) wurde untersucht, ob diese Patienten von der Therapie mit dem EGFR-Inhibitor profitieren können. Die prospektiv gewonnenen $\mathrm{Da}$ ten stammten von Tyrosinkinaseinhibitor-naiven Patienten mit NSCLC im Stadium IIIB-IV mit EGFR-Mutation. In der Intent-to-treat-Analyse aller Patienten mit ungewöhnlichen EGFR-Mutationen wurden verglichen: Gruppe $1 \mathrm{mit}$ Punktmutationen oder Duplikationen in Exon 18-21, Gruppe 2 mit de novo Exon20-Mutationen in Thr790Met alleine oder in Kombination mit anderen Muta- tionen und Gruppe 3 mit Exon-20-Insertionen. Von 600 Patienten, die in den Studien Afatinib erhalten hatten, wiesen 75 (12 \%) ungewöhnliche Mutationen auf. Dabei waren 38 der Gruppe 1, 14 der Gruppe 2 und 23 der Gruppe 3 zuzuordnen. Separat betrachteten die Forscher auch noch Patientengruppen mit den häufigsten ungewöhnlichen Mutationen (Gly719Xaa, Leu861Gln und Ser768Ile) alleine und in Kombination mit anderen Mutationen.

Objektiv sprachen 27 Patienten der Gruppe $1(71,1 \%)$ an, 2 der Gruppe 2 $(14,3 \%)$ und 2 der Gruppe 3 (8,7\%). Das mediane progressionsfreie Überleben lag in Gruppe 1 bei 10,7, in Gruppe 2 bei
2,9 und in Gruppe 3 bei 2,7 Monaten. Das mediane Gesamtüberleben unterschied sich ebenfalls deutlich in den 3 Gruppen mit 19,4 Monaten in Gruppe 1, 14,9 Monaten in Gruppe 2 und 9,2 Monaten in Gruppe 3. Die objektiven Ansprechraten bei den untersuchten häufigsten Mutationen lagen relativ hoch mit $77,8 \%$ bei Gly719Xaa, $56,3 \%$ bei Leu861Gln und $100,0 \%$ bei Ser768Ile.

Fazit: Afatinib ist bei verschiedenen selteneren EGFR-Mutationen aktiv, kaum dagegen bei de novo Thr790Met und Exon-20-Insertion. Damit könnte man Patienten mit ungewöhnlichen EGFRMutationen eine maßgeschneiderte Therapie vorschlagen. Friederike Klein

Yang JCH et al. Clinical activity of afatinib in patients with advanced non-small-cell lung cancer harbouring uncommon EGFR mutations: a combined post-hoc analysis of LUX-Lung 2, LUXLung 3, and LUX-Lung 6. Lancet Oncol. 2015; 16(7):830-8. 